\title{
Design and Implementation of a Hydrological Safety Warning System Based on ZigBee
}

\author{
Sai Cen ${ }^{1}$, Fei $\mathrm{Xu}^{2,{ }^{*}}$ and Meng Gui ${ }^{3}$ \\ ${ }^{1}$ College of Information Engineering, Wuhan University of Technology, Wuhan, China \\ ${ }^{2}$ Information Technology Research Institute, Wuhan University of Technology, Wuhan, China \\ ${ }^{3}$ Wuhan city Luqiao charge management center, Wuhan, China
}

Keywords: Flood Disasters, Hydrological Safety Warning, ZigBee Network, Mergency Refuge

\begin{abstract}
In recent years, there are many reports about the flood disaster. These reports reflect the lag of the early warning system of hydrological safety in our country, and it is an urgent need to establish the flood disaster safety early warning system of hydrological in these areas . In order to quickly aware of the hydrological changes before the flood disaster, we designed a hydrological safety warning system based on the ZigBee. Based on the hydrology sensor, the RS232 serial port will transmit the data to the ARM6410, after processing, and then through the ZigBee network transmission to the PC (personal computer), so that people observed real time hydrological security information, And the system can predict future hydrological conditions according to the current trend of hydrological data, when an exception occurs in a warning. so that the relevant departments can in pre-disaster emergency hedge, Avoiding causing casualties as many as possible.
\end{abstract}

\section{Introduction}

China is vast in territory, the rainfall distribution is different, especially in the south, many areas will be hit by heavy rain, and many low-lying areas are very prone to flood disaster in summer, The annual flood disaster has brought huge losses to the economic development and people's property security in these areas. However, due to the risk and complexity of the field environment is not suitable for the establishment of monitoring stations, therefore, it is very important to design a stable and reliable hydrological safety early warning system. In recent years, the construction and technology of hydrological information early warning system in China have made great progress, The hydrological safety early-warning system has provided the data for our country's geological disaster warning, But there is still a big gap with the western developed countries, especially the technology of Information acquisition and transmission is backward, which can not meet the real - time, fast and accurate requirements of hydrological safety early warning system. The hydrological safety early warning system designed by us can upload the data of each monitoring point to the monitoring center in real time.

\section{System Design and Implementations}

\section{A. Principle of Hydrological Safety Waring System}

Hydrological safety early warning system is mainly composed of hydrological data acquisition and safety management. Hydrological data acquisition consists of four functional modules, including hydrological sensor, ARM control part, ZigBee communication, network communication. The sensor is used to collect hydrological data, ARM used to obtain data from the sensor, and fream the data into a specific format, and then transmitted through the serial port to the ZigBee module, ZigBee module is used to upload data to the Coordinator. Network communication module is responsible for uploading the data to the computer. Security early warning management module consists of three parts,including basic information management, real-time monitoring and early warning management. 
Its function is to process the data, and Send an alarm when a data anomaly is found.Hydrological safety warning system structure shown in Figure 1.

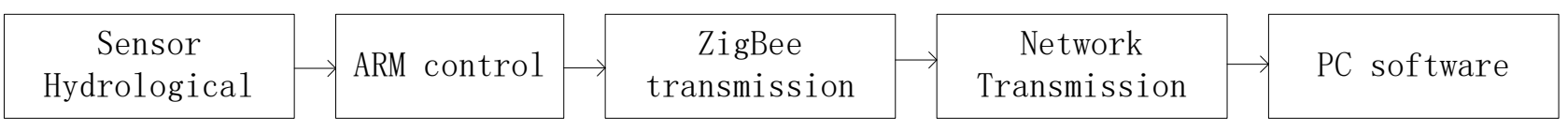

Fig.1 Structure diagram of hydrological safety warning system

\section{B. Hydrological Data Acquisition System Hardware Design}

\section{1)Hydrological Data Acquisition}

Flood disaster is a common geological disaster, A large number of studies have shown that $90 \%$ of the flood disasters caused by heavy rainfall. water level, water flow rate and other hydrological information is also a great relationship to the formation of flood disasters, so we prepare to collect rainfall and water levels and other hydrological data. The hydrological data collected by the hydrological sensor, including rainfall sensor, temperature sensor, water level sensor, humidity sensor and other sensors [2]. The hydrology acquisition sensor is installed in the collection site, powered by solar energy. It can convert the change to electrical signal When the hydrological information changed. These signals are transmitted to ARM via RS232 serial port. We use RealARM6410 as processor, The RealARM6410 development board is a high-performance, high integration, extensive expansion of the integrated development board introduce developed by Hua Tianzheng Technology [3].

\section{2)ZigBee Communication and Network Transmission Module Hardware Design}

ZigBee module independent designed by us based on TI's CC2530 chip. The CC2530 is a truely System on a chip solution for 2.4-GHz IEEE802.1, ZigBee and RF4CE applications [3],It can build powerful network nodes at very low cost. The communication distance of CC2530 can reach 200m without using the internal amplifier module. In order to expand the range of communication we used the amplifier chip RFX2041C.The communication distance of CC2530 can reach 2000m after using it. C. Hydrological Data Acquisition Software Design

\section{1)ZigBee Communication Module Software Design}

The system developed based on TI's Z-Stack, The Coordinator waits for the Router and Enddevice to join the network after initialization. The Router and Enddevice Perform two tasks after joining the network from the node. On the one hand they upload the real-time data collected by the sensor. On the other hand they have to upload a heartbeat package let the coordinator know that they are still alive. PC determine whether the sensor node dropped or failure by the heartbeat package.

ZigBee transmission module software flow chart shown in figure 2. The Coordinator is initialized after startup, and then starts the network. When initialization is complete, the Coordinator enters listening mode. Once it listens to the request from the node to join the network and then respond to the request, establish a network connection with the node. The Router queries weather there is networkt has been built after initialization, and will request to join the network if finds one, It also allows other nodes as children to join the network. The End device is similar to Router, But it do not allow other nodes as their child. Since there are many nodes uploading data at the same time, there may be conflicts between them. To solve this problem, we set up a" time window model", That is to say, the master node will send a short random time after receiving the data upload application, the children will sleep at this time, and upon data at the end of the time. so as to reduce the conflict between nodes, enhance network stability. Since all the nodes except Coordinate are powered by solar cells, in order to reduce power consumption, each node enter low-power sleep mode after upon data, and it will stop all communication activities until the next time you need to upload data.

Network transmission module is based on the chip of W5200, The W5200 chip is a Hardwired TCP/IP embedded Ethernet controller that enables easier Internet connection for embedded systems. 
It is possible to use 8 independent hardware sockets simultaneously. SPI (Serial Peripheral Interface) is provided for easy integration with the external MCU. In order to reduce power consumption of the system, W5200 provides WOL (Wake on LAN) and low power mode.

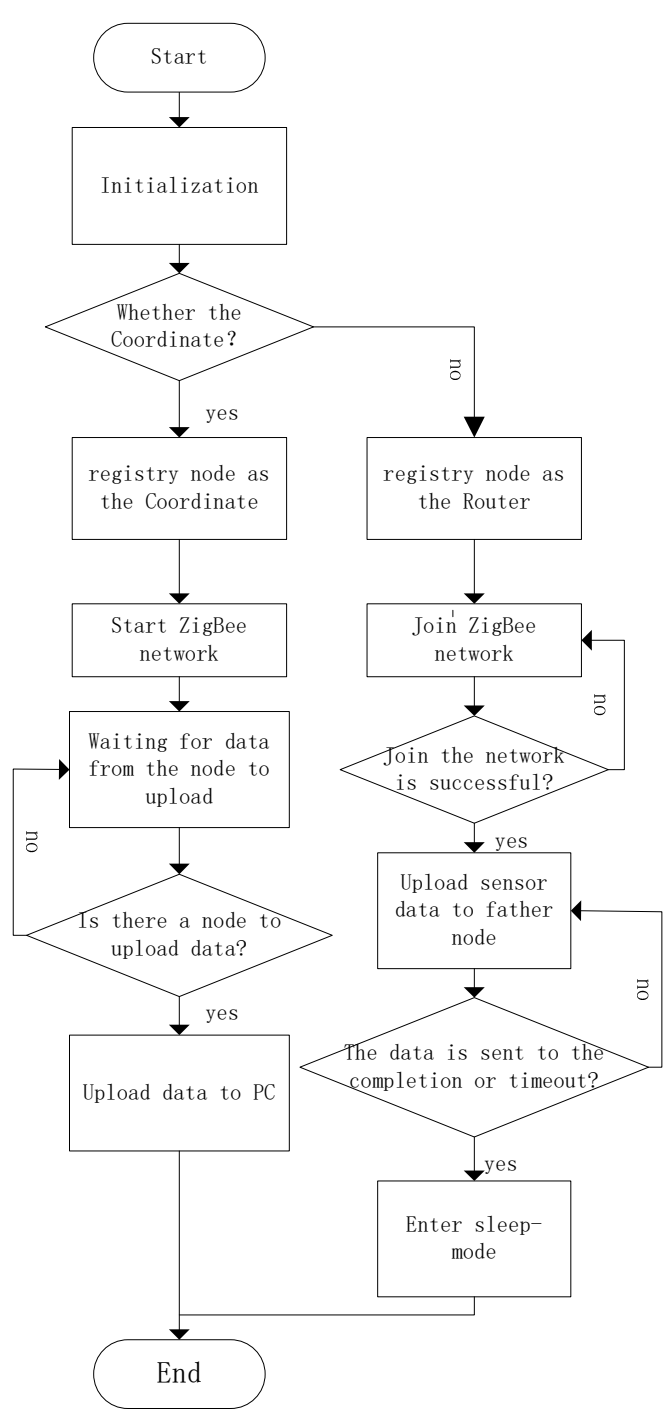

Fig.2 software flow chart of ZigBee transmission module

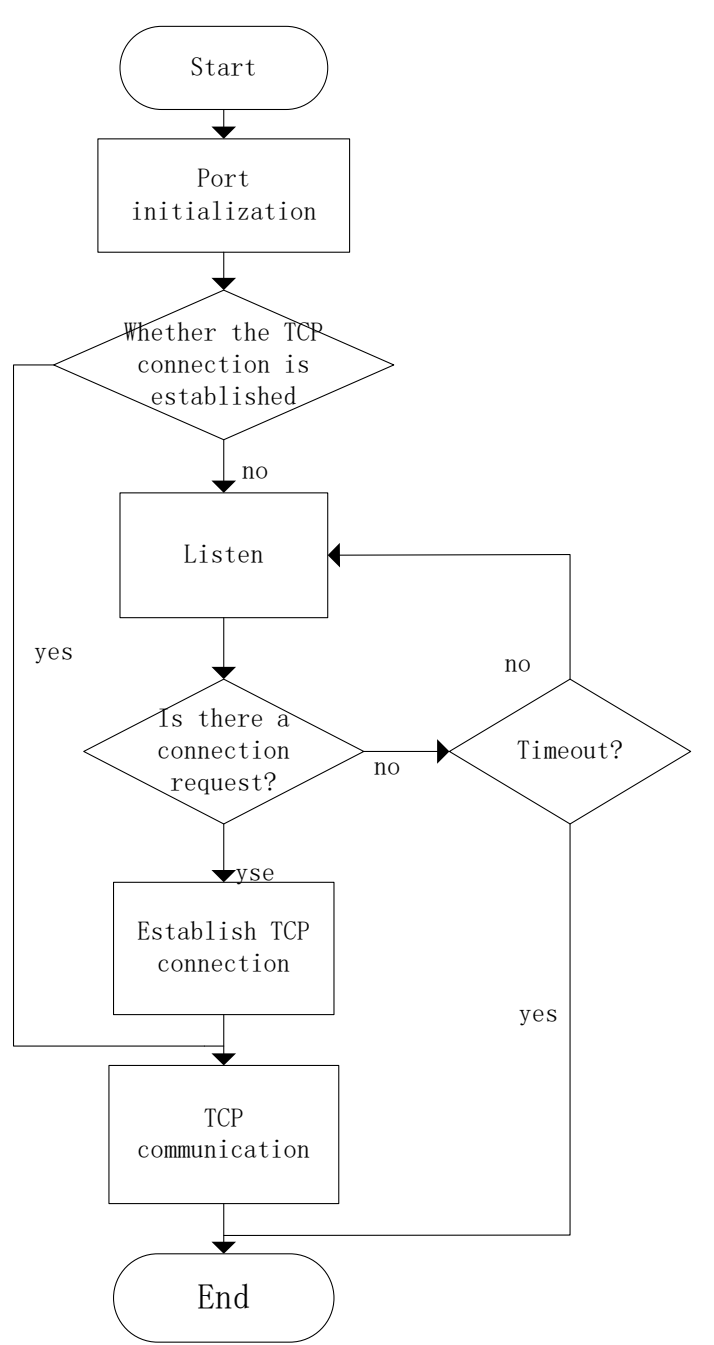

Fig. 3 software flow chart of network communication module

\section{2)Network Communication Module Software Design}

The Coordinate will upload data through W5200 controlled by SPI to PC, after receives the data sent from other node. When PC receives the data, it will responds to an acknowledgement frame, If the Coordinate does not receive the acknowledgement frame it will think that the data upload failed and upload the last record again.

The network communication module uses client, server mode for TCP / IP protocol communication, The TCP server is first initialized before communication, and will detect the connection state after initialization, if it already connected the Coordinate will upload data directly, if there is no connection it will enter the listening (listen) mode, waiting for the client to send a TCP connection request. Network communication module software flow chart shown in figure 3.

\section{3)The Design of Data Processing Software for Hydrological Safety Early Warning System}

The development software of data processing software for hydrological safety early warning system is Visual studio 2010, PC software is divided into four parts, including basic information management, communication, early warning and real-time display. The network communication, 
receives the data which the lower computer uploads. The real-time monitoring module will display the data of the network transmission module in the form of charts and so on. The basic information management module manages the update and query of the database, so it is very convenient to query the previous hydrological information. The monitoring and warning module predicts the trend of hydrological information by means of mathematical statistics, and alert when the emergencies happen. It can provide strong support for the early warning of geological disasters. The computer software function structure frame figure as shown in Figure 4.

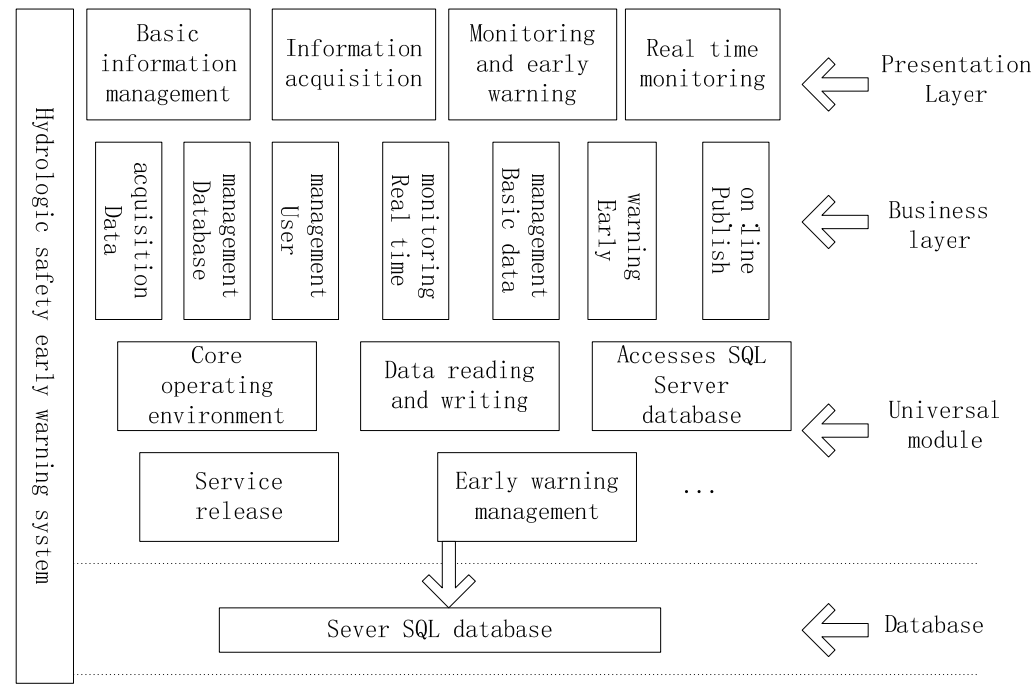

Fig. 4 software structure of hydrological safety early warning system

\section{(1) Basic Information Management of Hydrological Safety Early - warning System}

The basic information management system mainly has the user role management and the jurisdiction management as well as the software establishment function and so on. User roles are mainly administrators, employees, general users. The permissions of different roles are also different. The administrator has all the rights, including adding and deleting users, modifying user rights, viewing and modifying database data. Workers have the ability to view the background database data, but not modify permissions. Ordinary users can only view real-time data.

\section{(2) Information Processing of Hydrological Safety Early Warning System}

Heavy rainfall is one of the most important factors in the occurrence of flood disasters. Observation of rainfall for a hydrological monitoring point is an important aspect of disaster prediction. In general, the meteorological department's rainfall forecast is a large-scale forecast of a region, but in reality, the distribution of rainfall is limited and non-uniform. Thus, the specific regional disaster monitoring points should be arranged combining with on-site rainfall monitoring, so as to meet the needs of disaster prediction.

The rainfall node collects every rainfall and has its own trigger interrupt when it works, which records its own time point every time the rainfall is measured. Rainfall a few days before the disaster has also had an important impact on the disaster. The effective rainfall on the day of the disaster can be calculated by the power series, the calculation is as follows.

$$
R_{c}=R_{0}+\sum_{i=1}^{N} \alpha^{i} R_{i}
$$

Where Rc is the effective rainfall, and $\mathrm{R} 0$ for the day rainfall. $\mathrm{Ri}$ is for the former i days of rainfall, and $\alpha$ is the attenuation coefficient. When the selected coefficient is 0.8 , we can see that the closer the rain, the larger the impact of the current effective rainfall. 
According to the provisions of the National Weather Bureau, the effective rainfall within one day is divided into: light rain $1-10 \mathrm{~mm}$, moderate rain $10-25 \mathrm{~mm}$, heavy rain $25-50 \mathrm{~mm}$, heavy rain $50-100 \mathrm{~mm}$, heavy rain $100-200 \mathrm{~mm}$, heavy rain $200 \mathrm{~mm}$. These rainfall grades are closely related to the occurrence of flood disasters. The greater the effective rainfall, the greater the possibility of flooding.

$$
\begin{gathered}
Y_{X}=a_{0}+a_{1} X \quad a_{0}=\left(\sum Y_{i}\right) / m-\left(a_{1} \sum X_{i}\right) / m \\
a_{1}=\left[\sum X_{i} Y_{i}-\left(\sum X_{i} \sum Y_{i}\right) / m\right] /\left[\sum X_{i}^{2}-\left(\sum X_{i}\right)^{2} / m\right]
\end{gathered}
$$

Hydrological safety anomaly warning is to use the current data collected to calculate the next data using the least squares method.

$\mathrm{YX}$ is the estimated effective rainfall amount, and $\mathrm{m}$ is taken as the sample capacity, that is, the sample volume of the rainfall parameter. $\mathrm{Xi}$ and $\mathrm{Yi}$ are the arbitrary set of rainfall time $\mathrm{X}$ and the current effective rainfall value $\mathrm{Y}$ respectively.

\section{(3) Hydrological Safety Anomaly Warning}

When entering the monitoring system, as shown in Figure 5, you can clearly see the changes and forecasts of the various parameters. When one of the parameters of real-time data or forecast data exceeds the critical value, then an alarm is issued indicating that the staff member data is abnormal.

Hydrological safety early warning system
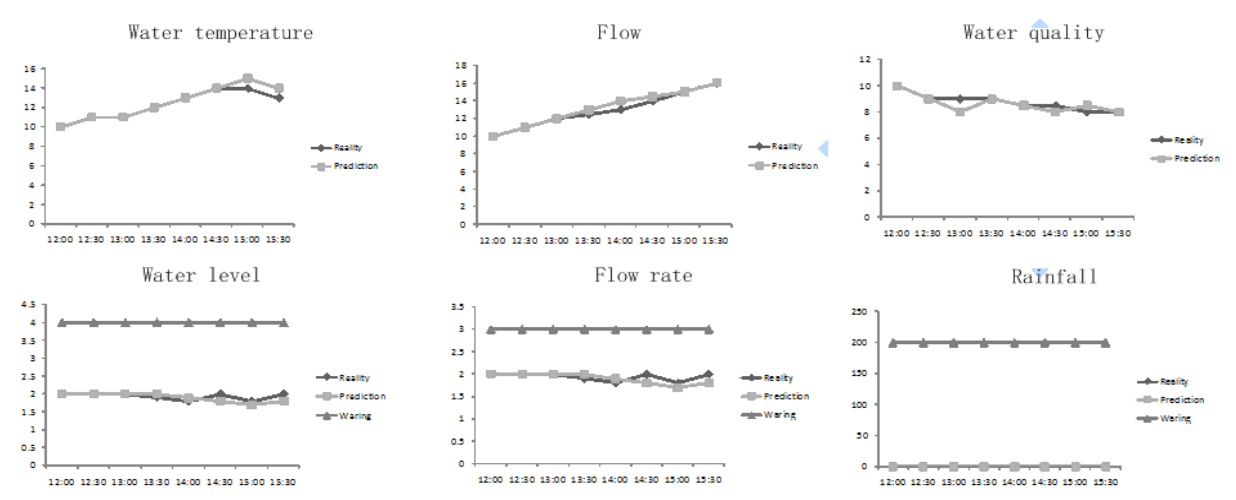

Fig.5 Data monitoring of hydrological safety pre-warning system

\section{Conclusions}

In this paper, a set of solar battery-powered hydrological safety early warning system based on ZigBee is designed. According to ZigBee's adaptive networking, the wireless nodes can upload the data collected by the sensor to the PC through distributed collaboration. It can be seen from the test that the system performance is stable and packet loss rate is small. Each node can enter the low-power mode after transmitting the data. It can effectively reduce the energy consumption of each node. PC-side software can process the collected data to predict future hydrological data and an alert is issued when data is abnormal. Stable and reliable realization of the hydrological safety early warning function. Stable and reliable hydrological safety early warning function has been realized.

\section{References}

[1]Daoxun Feng. Application of ZigBee and GPRS technology in Wireless Hydrological monitoring system [D]. Taiyuan: Taiyuan University of Technology, 2008 
[2]Liming Sun,JianZhong Li,yu Chen.Wireless sensor networks [M]. Beijing:Tsinghua University press, 2005

[3]XiaoLong Yuan. Embedded network video monitoring system based on ARM6410 [J]. Beijing: Beijing Jiaotong University, 2012

[4] LiTing Cai,PinHua Chen. Design of ZigBee data acquisition system based on CC2530 [J].ShanXi:Computer science and development, 2012

[5] Malek, Li,Chun Lin. Performance analysis of improving ZigBee wireless network using AODV mesh routing model[C]. 3rd International Conference on Materials Science and Information Technology, MSIT 2013

[6]Wen Xia,GuoWei Feng.Wu Xia. Design of power quality remote monitoring system based on W5200 [J].Being Jing: Electrical technology,2012

[7]CongPeng Zhang,KangKang Zhao. Development of serial port server system based on STM32 [J].ShenYang: Instrument technique and sensor,2011 